\title{
Exosomes derived from IL-12-anchored renal cancer cells increase induction of specific antitumor response in vitro: A novel vaccine for renal cell carcinoma
}

\author{
YAO ZHANG ${ }^{1}$, CHUN-LI LUO ${ }^{2}$, BAI-CHENG HE ${ }^{3}$, JIA-MO ZHANG ${ }^{1}$, GANG CHENG ${ }^{1}$ and XIAO-HOU WU ${ }^{1}$ \\ ${ }^{1}$ Department of Urology, The First Affiliated Hospital, ${ }^{2}$ Department of Laboratory Medicine, \\ ${ }^{3}$ Pharmaceutical College, Chongqing Medical University, Yi Xue Yuan Road, Chongqing, P.R. China
}

Received August 14, 2009; Accepted October 10, 2009

DOI: 10.3892/ijo_00000484

\begin{abstract}
Exosome-based immunotherapy for cancer holds promise, but needs improvements, especially for tumorderived exosomes. We investigated, whether exosomes derived from IL-12-anchored human renal cancer cells could enhance their immunogenicity and increase induction of specific antitumor response. A mammalian co-expression plasmid of glycolipid-anchored-IL-12 (GPI-IL-12) was constructed by subcloning IL-12A chain gene (P35 subunit) and a fusion gene containing GPI-anchor signal sequence of human placental alkaline phosphatase-1 (hPLAP-1) and IL-12B chain gene (P40 subunit) in pBudCE4.1. Then exosomes were prepared from renal cancer cells modified to express GPI-IL-12. The results showed that exosomes derived from IL-12-anchored renal cancer cells expressed renal cell carcinoma-associated antigen G250 and GPI-IL-12. The incorporation of GPI-IL-12 onto exosomes (exosomesGPI-IL-12, EXO/IL-12) significantly promotes proliferation of $\mathrm{T}$ cells, and subsequently increased the release of IFN- $\gamma$. Notably, stimulation with EXOs/IL-12 could efficiently induce antigen-specific cytotoxic T lymphocytes (CTLs), resulting in more significant cytotoxic effects in vitro. These results suggested that exosomes derived from IL-12anchored renal cancer cells bore GPI-IL-12 and G250, which have tumor rejection antigen with enhanced immunogenicity and antitumor effects, representing a novel strategy of exosomes-based vaccine for renal cell carcinoma.
\end{abstract}

\section{Introduction}

Renal cell carcinoma (RCC) accounts for approximately 2-3\% in all adult malignancies. Incidence rates for renal cell cancer

Correspondence to: Professor Xiao-Hou Wu, Department of Urology, The First Affiliated Hospital, Chongqing Medical University, Yi Xue Yuan Road, Chongqing, P.R. China

E-mail:wuxiaohou80@hotmail.com

Key words: renal cell carcinoma, exosomes, interleukin-12, tumor vaccine, immunotherapy have been increasing steadily each year in Europe and the United States in the last three decades (1). Treatment of metastatic RCC is still challenging because of its resistance to conventional therapies, such as radiation or chemotherapy (2). Encouragingly, RCC is one of the most immunoresponsive of human malignancies. Thus, various immunotherapeutic strategies have been used to increase the antitumor immunity, such as the administration of cytokines, tumor cell-based vaccines, dendritic cell (DC)-based vaccines (3). However, the application of cytokines and existing tumor vaccines are often only moderately tolerated, and the response rates remain unsatisfactory (4). Therefore, new therapeutic vaccine needs to be developed for RCC treatment.

Exosomes (EXOs), 30-100 nm microvesicles secreted by many types of cells, had not received extensive attention until Raposo et al for the first time reported that B cellderived exosomes induced antigen-specific MHC class Irestricted $\mathrm{T}$ cell responses in 1996 (5). The biology of exosomes derived from tumor cells (TEXs) has recently raised much interest due to their bearing MHC-I molecules, tetraspanins, HSP70-80, LAMP1, as well as tumor rejection antigens, and triggering MHC-I-restricted T-cell responses and antitumor immunity (6-10). However, in recent years, proteomics studies have found that exosomes also expressed immunosuppressive molecules, which reduced the immunogenicity of the exosomes and inhibited activation of lymphocytes by suppressing the expression of CD3- $\zeta$ and downregulated the JAK3-STAT5 signal pathways $(11,12)$. However, the immunogenicity of TEXs is limited. Therefore, novel ways need to be explored to enhance the immunogenicity of TEXs.

Interleukin 12 (IL-12) is a critical cytokine produced by phagocytic cells and antigen-presenting cells (APCs) and it plays an essential role in induction of cellular immune activity (13). IL-12 can promote the killing capacity of NK and cytotoxic T cells and upregulates the level of Jak-STAT signaling intermediates in both tumor and immune effector cells (14), drives the differentiation of Th0 to Th1 and strongly induces IFN- $\gamma$ production (15). However, due to its very short half-life and side-effects of systemic administration, in recent years, more research has been done on adjuvant vaccines (16). Previous studies confirmed that GPI-IL-12 expressed on the tumor cell surface could be effective in 
inducing antitumor immune response, and GPI-anchored cytokines expressed on the tumor cell surface might be an alternative approach to deliver cytokines at the immunization site during vaccination against cancer (17). Exosomes, coated by two-layer lipid membrane, just like cell membrane structure, can be easy to anchor with GPI-anchored proteins (18). Therefore, we speculate that GPI-IL-12 might be a good way to enhance the immunogenicity of TEXs and exosomebased immunotherapy effects.

Our pre-experimental study for the first time found that renal cancer-derived exosomes highly expressed RCCassociated antigen G250. With high cell surface expression in RCC and limited expression in normal tissue, G250 serves as an excellent therapeutic target (19). Exosomes expressing G250 was a necessary prerequisite for developing renal cancer vaccine. In the present study, we have investigated whether exosomes derived from IL-12-anchored renal cancer cells could enhance its immnongenicity and increase the induction of specific antitumor response. For this purpose, we constructed mammalian co-expression plasmid of pBudCE4.1/IL-12A/IL-12B-GPI and obtained stable transfectants of renal cancer cells, and isolated the exosomes from the culture supernatants of the stable cell line. We found that exosomes derived from IL-12-anchored renal cancer cells bore GPI-IL-12 and G250, could significantly enhance their immunogenicity and efficiently induce antigen-specific immune response and CTL, resulting in more significant cytotoxic effects in vitro. Therefore, the exosome-based vaccine might be a novel strategy for treatment of renal cancer.

\section{Materials and methods}

Cell line and reagents. Human renal cancer cell line RC-2 was purchased from Shanghai Cell Bank (China). Centriplus centrifugal ultrafiltration tube $(100 \mathrm{kDa})$, Amicon ultrafiltration centrifuge tube $(100 \mathrm{kDa})$, PVDF membrane were purchased from Millipore (CA, USA). Mouse anti-human HSP70, IL-12 monoclonal antibody, rabbit anti-human ICAM-1 monoclonal antibody, rabbit anti-human polyclonal antibody G250 were purchased from Santa Cruz (CA, USA). PCR product purification kit was purchased from ShanghaiHuashun Biotechnology Corporation (China). Restriction endonuclease, quick ligation kit, Primstar enzymes and other PCR reagents were purchased from Takara Corporation (Japan). E. coli DH5a strain was provided by Basic Medicine Institute of Chongqing Medical University (China). The plasmid of pBudCE4.1 was purchased from Invitrogen Corporation (USA). EST clone and pAdTraceTO4-IL-12BGPI plasmid were generously provided by Professor T.C. He, the University of Chicago, IL, USA.

Construction and identification of mammalian co-expression plasmid of pBudCE4.1/IL-12A/IL-12B-GPI. A mammalian co-expression plasmid of GPI-anchor-IL-12 was constructed by subcloning p35 cDNA (IL-12A) in pBudCE4.1 at KpnI/ XhoI sites and a fusion gene containing GPI-anchor signal sequence of hPLAP-1 and p40 cDNA (IL-12B) in the same plasmid at BamHI/XbaI sites. According to GeneBank sequences (P35 Accession AF180562), primers were designed to amplify IL-12A-chain gene fragment. The up- stream primer sequence (P1) was 5'-cggtaccaccatgggcatgt ggcccctgggtcagcctcc and the downstream primer sequence (P2) was 5'-cgctcgagttaggaagcattcagatagctcatc. PCR products of IL-12A-chain gene and plasmid pBudCE4.1 were digested with $K p n I$ and $X h o I$ and the purposed DNA fragments were recovered. IL-12A-chain gene fragment was cloned to pBudCE4.1 plasmid to construct pBudCE4.1/IL-12A plasmid. Plasmids of pBudCE41/IL-12A and pAdTraceTO4IL-12B-GPI were digested with BamHI and XbaI. Large fragments of pBudCE4.1/IL-12A and small fragments of pAdTraceTO4-IL-12B-GPI were recovered with gel and connection kit of Takara was used to build a co-expression plasmid pBudCE4.1/IL-12A/IL-12B-GPI. The recombinant plasmids were transformed into E. coli DH5a with $40 \mu \mathrm{g} / \mathrm{ml}$ of Zeocin for screening. Recombinant plasmids were identified with PCR amplification, restriction enzyme digestion and DNA sequencing.

Establishing transfectants expressing GPI-anchored IL-12. RC-2 transfectants expressing GPI-IL- 12 were established by transfecting pBudCE4.1/IL-12A/IL-12B-GPI plasmid by Lipofectamine ${ }^{\mathrm{TM}} 2000$. RC-2 cells were inoculated in the special FBS RPMI-1640 medium until 80\% cells confluence, cells transfected with empty plasmid and non-transfected cells as controls. Stable cells line was screened by Zeocin. About $100 \mathrm{ml}$ of stable cell supernatant was collected for exosome preparation.

Preparation of exosomes. The RC-2 cell-derived exosomes were isolated and purified as described (20). Culture supernatants $(100 \mathrm{ml})$ were collected and sequentially centrifuged $\left(4^{\circ} \mathrm{C}\right)$ at $300 \mathrm{x} \mathrm{g}$ for $10 \mathrm{~min}, 800 \mathrm{x} \mathrm{g}$ for $30 \mathrm{~min}$ and $10,000 \mathrm{x} \mathrm{g}$ for $30 \mathrm{~min}$. The clarified supernatant was concentrated by centrifugation at $1000 \mathrm{x} \mathrm{g}$ for $30 \mathrm{~min}$ in a prerinsed $100 \mathrm{kDa}$ MWCO Centrifugal Filter Device and the concentrated exosomes were collected and resuspended in $20 \mathrm{ml}$ PBS. The ultracentrifuge supernatant was underplayed with $30 \%$ sucrose $/ \mathrm{D}_{2} \mathrm{O}$ density cushion, followed by ultracentrifuging at $100,000 \times \mathrm{g}$ for $60 \mathrm{~min}$. At the bottom, the cushion was collected and diluted in $10 \mathrm{ml}$ of PBS. The exosomes were further concentrated by centrifuging for $30 \mathrm{~min}$ at $1000 \mathrm{x} \mathrm{g}$ in prerinsed $100 \mathrm{kDa}$ MWCO Amicon ultra-15 to a volume of about $3 \mathrm{ml}$. Membrane filter $(0.22 \mu \mathrm{m})$ was used, after sterilization the exosome was stored at $-80^{\circ} \mathrm{C}$. Bradford method was used for quantification of the total protein of exosomes (EXO: exosomes derived from untransfected cells; EXO/ev: exosomes derived from empty plasmid transfected cells; EXO/IL-12: exosomes derived from transfected cells).

Transmission electron microscopy and Western blot analysis. Morphology of exosomes was identified with transmission electron microscopy. Exosomes $(20 \mu \mathrm{l})$ were resuspended and loaded on electron microscopy grids. Heavy metal staining was performed with $2 \%$ phosphotungstic acid ( $\mathrm{pH}$ 6.8) for $1 \mathrm{~min}$ and then observed by transmission electron microscopy. Pictures were analyzed with Digimizer Image Analysis Software (http://www.digimizer.com) to measure the diameter of exosomes. Western blot was used to identify their molecular composition and the expression of G250 and GPI-IL-12. Exosomal protein were extracted as described 
(21), and analyzed by Western blot. In brief, $50 \mu \mathrm{g}$ of total protein was loaded into each well of an SDS-gel for separation by PAGE, and then transferred onto PVDF membranes. The blotted membranes were blocked for $60 \mathrm{~min}\left(4^{\circ} \mathrm{C}\right)$ with TBST (0.25 M Tris, $\mathrm{pH} 7.5,150 \mathrm{mM}$ sodium chloride, and $0.2 \%$ Tween-20) containing 5\% skim milk, followed by probed overnight with primary antibody and horseradish peroxidase-coupled secondary antibody. After three washes with TBST, blotted proteins were detected using an image acquisition and analysis system. The following antibodies were used for the Western blots: anti-HSP70, anti-ICAM-1, anti-IL-12 and anti-G250 antibodies.

Confocal laser scanning microscopy and flow cytometry analysis. Cells were fixed with $4 \%$ paraformaldehyde and washed with PBS three times and blocked with $3 \%$ fetal bovine serum for $30 \mathrm{~min}$ at room temperature (RT), then incubated with mouse anti-human IL-12 antibody overnight at $4^{\circ} \mathrm{C}$. Followed by incubation with FITC labeled second antibody for $1 \mathrm{~h}$. The images were observed and obtained by confocal microscopy. Untransfected cells and empty plasmid-transfected cells served as controls. The GPI-linkage of IL-12 was confirmed by washing down RC-2-GPI-IL-12 cells with phosphatidylinositol-specific phospholipase C (PIPLC) for flow cytometry to detect the membrane-bound GPIIL-12 expression.

Proliferation assays. Peripheral blood mononuclear cells (PBMCs) were isolated from normal healthy donor as previously described (22). T cells were enriched from PBMC by plate adherent and isolated by Nylon Fiber Column T (Seebio Company, China). PHA-activated T cells were prepared using $5 \mu \mathrm{g} / \mathrm{ml}$ PHA (Sigma, Japan) by the standard procedure. PHA-activated cells were washed again and resuspended into RPMI-1640 medium at a concentration of $1 \times 10^{7}$ cells $/ \mathrm{ml}$. CFSE-based proliferation assays of $\mathrm{T}$ cells were performed as precisely described (23). Carboxyl fluorescein succinimidyl ester (CFSE) (Molecular Probes Inc., USA) was added to the cell suspension at the final concentration of $2.5 \mu \mathrm{M}$ and incubated for $10 \mathrm{~min}$ at $\mathrm{RT}$ in the dark. Labeling was terminated by adding the same volume of $50 \%$ fetal calf serum to quench the free CFSE for $5 \mathrm{~min}$ at RT. The labeled cells were then washed five times with sterile PBS containing 10\% FCS and resuspended into RPMI-1640 medium at $1 \times 10^{6} / \mathrm{ml}$. The labeled cells (responders) were cocultured with PBS $(100 \mu \mathrm{l})$, EXO $(10 \mu \mathrm{g} / 100 \mu \mathrm{l}), \mathrm{EXO} / \mathrm{ev}$ $(10 \mu \mathrm{g} / 100 \mu \mathrm{l}), \mathrm{IL}-12(80 \mathrm{pg} / 100 \mu \mathrm{l})$ or EXO/IL-12 $(10 \mu \mathrm{g} /$ $100 \mu \mathrm{l}$ ) for $48 \mathrm{~h}$ in five independent assays, respectively. Each group had triplicate wells. Cultured cells were then harvested, washed twice with PBS, then analyzed using a FACScan flow cytometer. FACS data analysis was performed using CellQuest software.

$I F N-\gamma$ release assay. PHA-activated T cells $\left(1 \times 10^{6}\right)$ (not dyed with CFSE) were co-cultured with PBS (100 $\mu 1)$, EXO $(10 \mu \mathrm{g} / 100 \mu \mathrm{l}), \mathrm{EXO} / \mathrm{ev}(10 \mu \mathrm{g} / 100 \mu \mathrm{l}), \mathrm{IL}-12(80 \mathrm{pg} / 100 \mu \mathrm{l})$ or EXO/IL-12 $(10 \mu \mathrm{g} / 100 \mu \mathrm{l})$ for $48 \mathrm{~h}$ in five independent assays as described in the proliferation assay. Supernatant was harvested and analyzed for the release of IFN- $\gamma$ to determine the IL-12-dependent T cell stimulation. Each group had

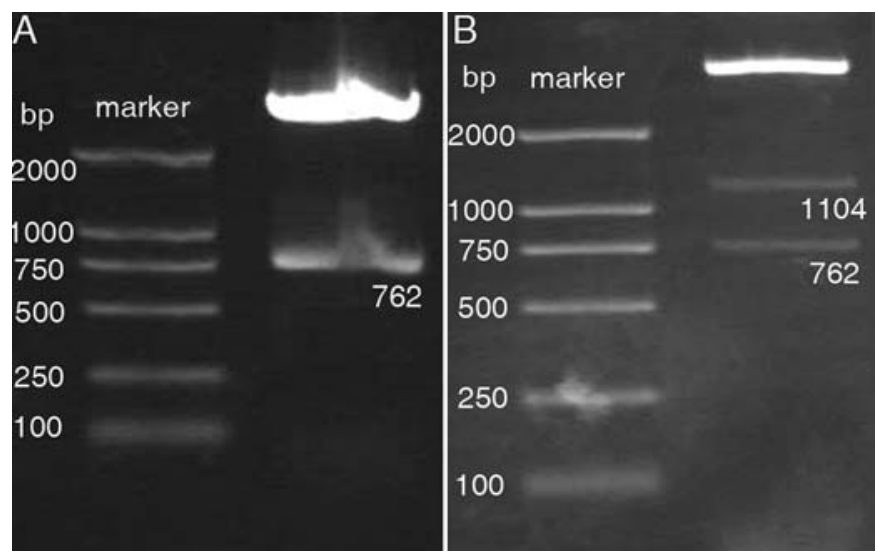

Figure 1. PCR amplification and identification of plasmids of pBudCE4.1/IL12A and pBudCE4.1/IL-12A/IL-12B-GPI. (A) The plasmid of pBudCE4.1/ IL-12A was digested with KpnI and XhoI, the band of 762 bp was the IL12A gene fragment; (B) The pBudCE4.1/IL-12A/IL-12B-GPI plasmid was digested with $K p n I$, XhoI, BamHI and XbaI, the bands of 762 and 1104 bp were gene fragments of IL-12A and IL-12B-GPI, respectively.

triplicate wells. IFN- $\gamma$ release was determined by sandwich ELISA (eBioscience, Inc., USA).

Cytotoxicity assays. Dendritic cells were isolated from human peripheral blood mononuclear for cytotoxicity assay. Adhered cells from PBMC were propagated in RPMI-1640 complete medium containing $1000 \mathrm{IU} / \mathrm{ml}$ recombinant human granulocyte macrophage colony stimulation factor (GM-CSF) and recombinant human interleukin 4 (IL-4) for 6 days. The morphology of DC was identified by SEM. Exosome-loaded DCs promoted activation of autologous $\mathrm{T}$ cells used as effector cells. Autologous DCs and T cells were co-inoculated with EXO/ IL-12 $(10 \mu \mathrm{g} / \mathrm{ml})$ for $48 \mathrm{~h}$ in a humidified $5 \% \mathrm{CO}_{2}$ incubator. PBS, IL-12, EXO or EXO/ev was used as control. Target cells were RC-2, T-24 (bladder cancer cell line) or SW480 (colon cancer cell line). Cytotoxicity assays based on PI costaining of CFSE-labeled target cells were performed precisely as described (24). In brief, target cells were labeled with $2.5 \mu \mathrm{M}$ CFSE, which strongly labels cells and allows differentiation between target and effector cells. Labeled target cells $(10,000)$ were coincubated in 24-well plates with given amounts of effector cells for $6 \mathrm{~h}$ in a humidified $5 \% \mathrm{CO}_{2}$ incubator. All cells were harvested and resuspended in PBS and analyzed by flow cytometry. Each group had triplicate wells. Killing rate was assessed by the percentage of PI- costaining cells out of gated CFSE-labeled cells. In all experiments the percentage of PI-positive CFSE-labeled cells in wells cultured without effectors did not exceed $15 \%$. Specific killing rate $=$ (experimental killing rate - natural death rate)/(100\% natural death rate); Experimental killing rate $=\left(\mathrm{CFSE}^{+} / \mathrm{PI}^{+}\right.$ target cells $) /\left(\mathrm{CFSE}^{+}\right.$target cells).

Statistical analysis. Statistical differences between two groups were analyzed by one-way analysis of variance (ANOVA) or Student's t-test. Data were recorded as mean $\pm \mathrm{SD}$, and values of $\mathrm{P}<0.05$ considered significant. 

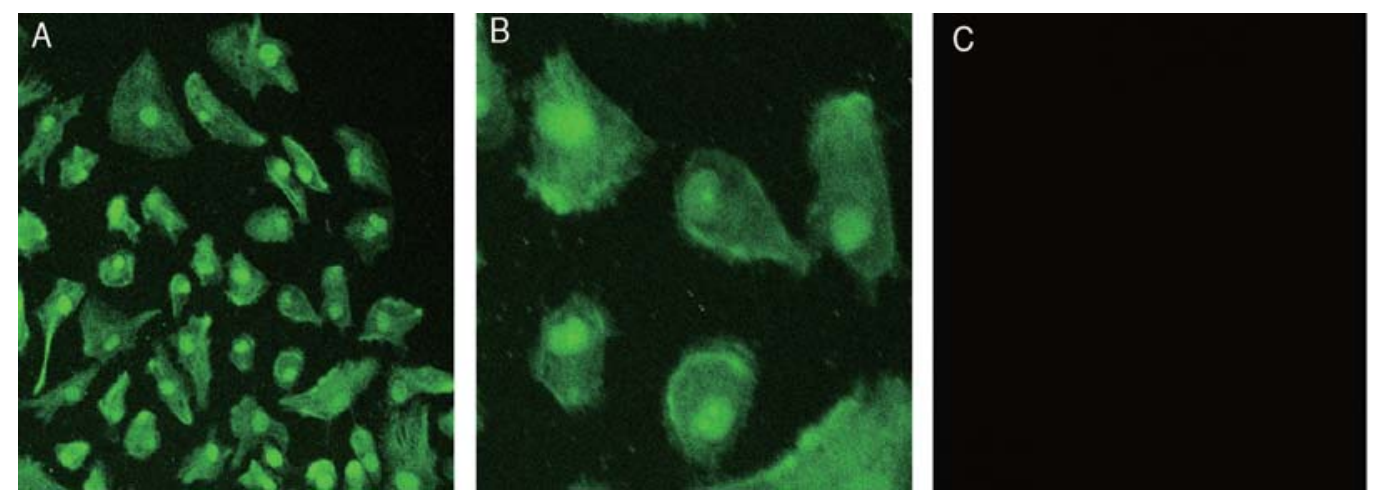

Figure 2. Confocal laser scanning microscopy observated the expression of GPI-IL-12 on cell surface of the stable transfectants. Cells were prepared and fixed with $4 \%$ paraformaldehyde, and immunostained with mouse anti-human IL-12 antibody overnight at $4^{\circ} \mathrm{C}$ before washed with PBS three times, followed by FITC-labeled second antibody for $1 \mathrm{~h}$ at $37^{\circ} \mathrm{C}$. (A and B) Positive expression, transfected group; (C) Negative expression.

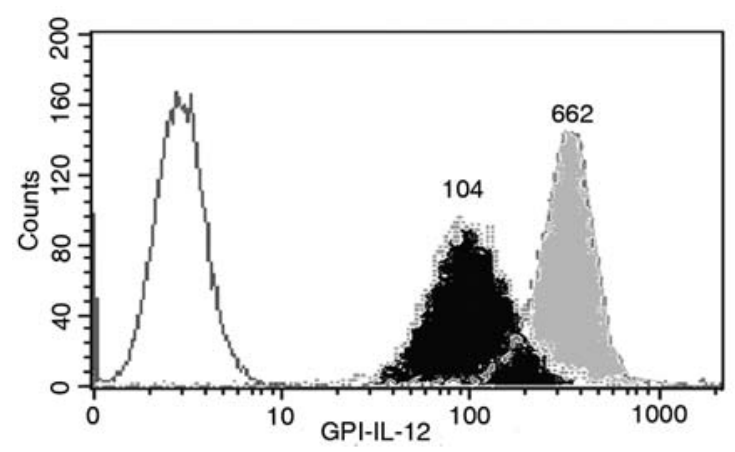

Figure 3. Chimeric GPI-IL-12 was expressed as GPI-anchored form. Cells were immunostained with anti-IL-12 mAb (filled histogram), followed by FITC-conjugated goat anti-mouse IgG. PI-PLC-treated cells were analyzed by flow cytometry. The values for mean fluorescent intensity for IL-12 expression are indicated. PI-PLC-treated cells show lower levels of cell surface expression of GPI-IL-12.

\section{Results}

Identification of mammalian co-expression plasmid. Plasmid pBudCE4.1/IL-12A was digested with $K p n I$ and XhoI, and resulted in band of IL-12A-chain (762 bp) (Fig. 1A). The correct sequence was identified by DNA sequencing (data not shown). The recombinant plasmids pBudCE4.1/IL-12A/ IL-12B-GPI were digested with KpnI, XhoI, BamHI and $X b a I$, and resulted in two bands, 1104 and 762 bp (Fig. 1B), named IL-12A-chain and IL-12B-GPI, respectively. The correct sequence was identified by DNA sequencing (data not shown), showing that co-expression plasmid of pBudCE4.1/IL-12A/IL-12B-GPI was successfully constructed.

IL-12 is expressed on the renal cancer cell surface as a GPIanchored protein. The results from confocal laser scanning microscopy observation indicated that IL-12 was mainly expressed on the membrane of the stable transfectants of RC-2 cells and also observed on the membrane of nucleus. No fluorescence intensity was observed in non-transfected group and empty vector group (Fig. 2). To determine whether the chimeric IL-12 on the cell surface was expressed as GPIanchored protein, RC-2-GPI-IL-12 cells were treated with
PI-PLC and analyzed by flow cytometry. PI-PLC-treated cells showed lower levels of cell surface expression of GPIIL-12, indicating that IL-12 was anchored to the cell surfaces via a GPI-moiety (Fig. 3).

Identification of exosomes derived from RC-2-GPI-IL-12 transfectants. An exosome can be identified by three major criteria (25): i) Morphology, exosomes have typical characteristic of 'saucer' or round morphology, coated by bi-layer lipid membrane; ii) size $30-100 \mathrm{~nm}$ in diameter; iii) protein composition. Exosomes derived from RC-2-GPI-IL-12 cells were successfully isolated with application of ultrafiltration and sucrose $/ \mathrm{D}_{2} \mathrm{O}$ gradient ultracentrifugation. Transmission electron microscopy (TEM) showed that exosomes were spherical vesicles with a mean $52.2 \pm 25.6 \mathrm{~nm}$ and surrounded by the two-layer lipid membrane. Exosomes (95\%) had a diameter between 30 and $80 \mathrm{~nm}$. The mean protein concentration of exosomes was $1.7 \pm 0.26 \mathrm{mg} / \mathrm{ml}$. Exosomes from $\mathrm{RC}-2$ cells without plasmid transfection and transfected with empty vector were also prepared, respectively, and visualized under TEM. There were no significant difference in morphology and size among these exosomes.

To testify whether GPI-IL-12 expressed on exosomes derived from the RC-2-GPI-IL-12 transfectants and whether GPI-IL-12 could influence the protein components of exosomes, we compared the protein pattern of exosomes derived from cells of three groups (transfected group, non-transfected group and empty vector group) by Western blot. As shown in Fig. 4C, GPI-IL-12 could be detected in exosomes of transfection group, but not in others. There was not significant difference among the protein expression of HSP70, ICAM-1 and G250 on exosomes derived from cells of three groups. The amount of IL-12 was $80 \pm 9.6 \mathrm{pg} / \mathrm{ml}$ in $10 \mu \mathrm{g}$ of exosomes, which was quantified by ELISA assay. Those results showed that exosome-GPI-IL-12 could be obtained from IL-12anchored renal cancer cells and IL-12 did not influence the protein components of exosomes.

Augmentation of activated T-cell proliferation by exosomesGPI-IL-12. To determine whether exosomes isolated from RC-2-GPI-IL-12 cells could induce proliferation of activated $\mathrm{T}$ cells more efficiently, we measured the proliferation rate of 

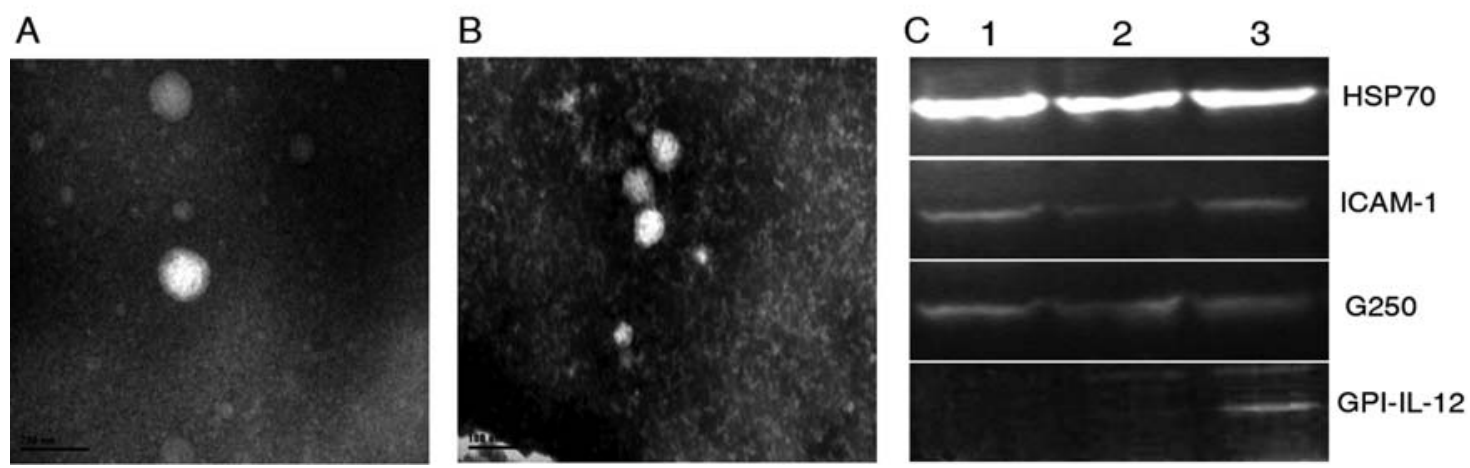

Figure 4. Identification of tumor-derived exosomes. Exosomes were isolated from RC-2 cells transfected with GPI-IL-12 gene or from RC-2 cells without gene transfection, and then visualized under TEM. They were spherical vesicles surrounded by the two-layer lipid membrane. The mean diameter was $52.2 \pm 25.6 \mathrm{~nm}$ and $95 \%$ of them distributed between 30 and $80 \mathrm{~nm}$. (A) Exosomes derived from gene-modified renal cells, bar $=200 \mathrm{~nm}$. (B) Conventional exosomes, bar $=100 \mathrm{~nm}$. (C) Western blot analyzed protein composition of exosomes. Equal amount of exosomal protein derived from 1 (non-transfeced group), 2 (empty vector-transfected group) and 3 (transfected group), the primary antibodies used were anti-HSP70, anti-ICAM-1, anti-G250 and anti-IL-12 antibodies.

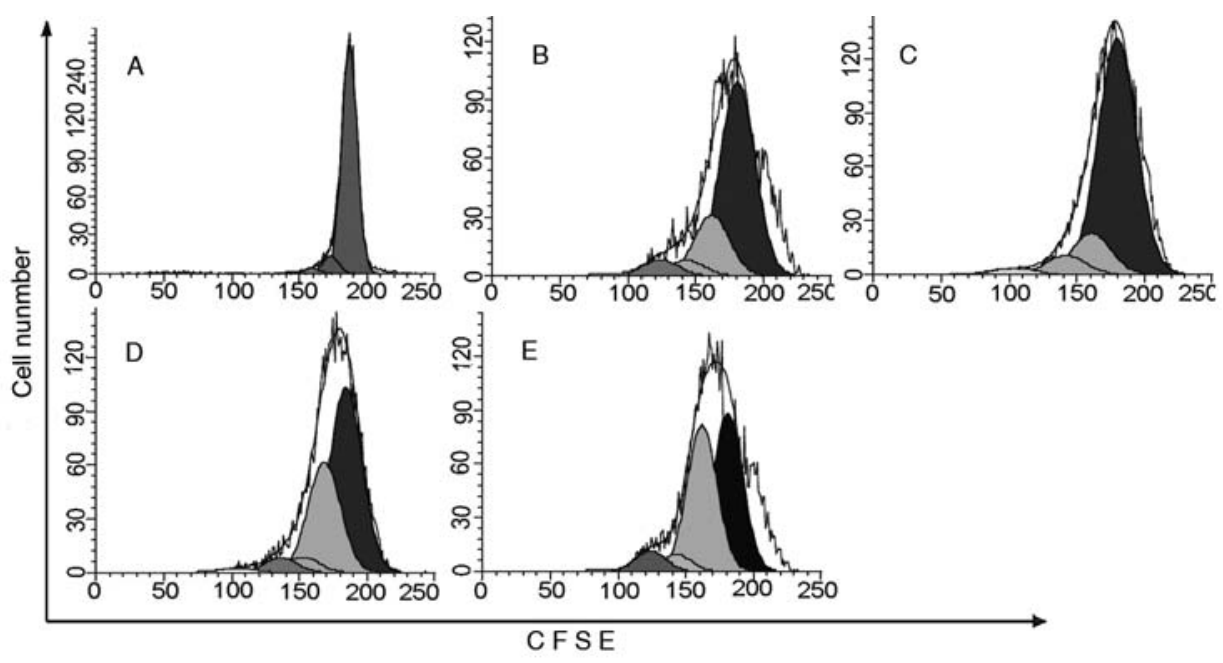

Figure 5. EXO/IL-12 induces proliferation of T cells. PHA-activated T cells were stained with CFSE $(2.5 \mu \mathrm{M})$ for 10 min at $37^{\circ} \mathrm{C}$ and resuspended into RPMI-1640 medium at a concentration of $1 \times 10^{6} \mathrm{cells} / \mathrm{ml} / \mathrm{well}$ at 24 -well plates, following with stimulation of EXO $(10 \mu \mathrm{g}), \mathrm{EXO} / \mathrm{ev}(10 \mu \mathrm{l}), \mathrm{IL}-12(80 \mathrm{pg})$, EXO/IL-12 $(10 \mu \mathrm{g})$ or PBS for $48 \mathrm{~h}$ in five independent assays. The labeled cells were collected and analyzed the proliferation rate by FACScan flow cytometer. FACS data analysis was performed using CellQuest software. Values are presented as mean \pm SD of triplicate wells. (A) Stimulated by PBS; (B) Stimulated by EXO; (C) Stimulated by EXO/ev; (D) Stimulated by IL-12; (E) Stimulated by EXO/IL-12.

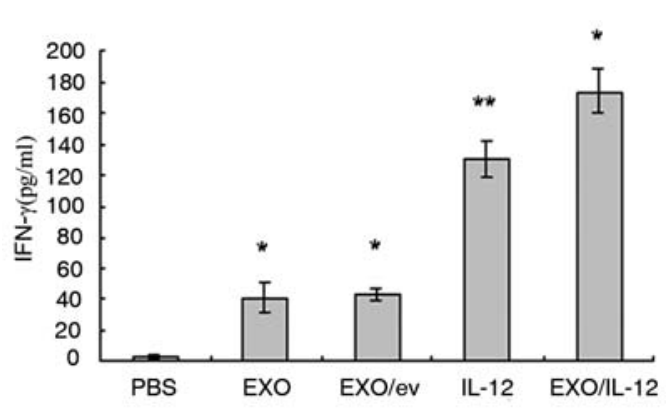

Figure 6. EXO/IL-12 induced the release of IFN- $\gamma$ by T cells. T cells were isolated from PBMC and activated by PHA $(5 \mu \mathrm{g} / \mathrm{ml})$ for $72 \mathrm{~h}$, and then cocultured with PBS, EXO, EXO/ev, IL-12 or EXO/IL-12, respectively. The supernatants were collected after $48 \mathrm{~h}$ and the release of IFN- $\gamma$ were determined by ELISA. Values are represented as mean $\pm \mathrm{SD}$ of triplicate wells. EXO or EXO/ev compared with PBS; IL-12 compared with EXO or EXO/ev; EXO/IL-12 compared with IL-12. ${ }^{*} \mathrm{p}<0.05,{ }^{* *} \mathrm{p}<0.01$. activated T cells stimulated with PBS, EXO, EXO/ev, IL-12 or EXO/IL-12, respectively, in the proliferation assay by flow cytometry. As shown in Fig. 5, Table I, the proliferating reaction of $\mathrm{T}$ cells was significantly enhanced by stimulating with EXO, EXO/ev, IL-12 or EXO/IL-12 alone compared with PBS. The highest proliferating reaction was observed in $\mathrm{T}$ cells stimulated with EXO/IL-12 group. These findings indicated that GPI-IL-12-expressed exosomes were functional in inducing T-cell proliferation.

Exosomes-GPI-IL-12 increased induction of the release of IFN- $\gamma$ by activated T cells. IL-12 has been shown to stimulate the proliferation of $\mathrm{T}$ cells and induce the release of Th1 type cytokines such as IFN- $\gamma$ (4). Therefore, the ability of EXO/ IL-12 to induce the release of IFN- $\gamma$ was determined in IFN- $\gamma$ release assay by ELISA. The results demonstrated that EXO, EXO/ev, IL-12 and EXO/IL-12 induced lymphocytes to secret IFN $-\gamma$. The amount of IFN $-\gamma$ was $40.7 \pm 9.5$ and 
Table I. Proliferation rate (\%) of generations of T cells after stimulation by PBS, EXO, EXO/ev, IL-12 or EXO/IL-12. ${ }^{\mathrm{a}}$

\begin{tabular}{|c|c|c|c|c|c|}
\hline Value (\%) & PBS & EXO & $\mathrm{EXO} / \mathrm{ev}$ & IL-12 & EXO/IL-12 \\
\hline Parent & $89.3 \pm 2.1$ & $71.2 \pm 3.9$ & $72.9 \pm 5.4$ & $60.7 \pm 0.8$ & $44.0 \pm 3.4$ \\
\hline G2 & $7.8 \pm 1.2$ & $15.2 \pm 6.7$ & $17.2 \pm 6.5$ & $30.1 \pm 3.3^{\mathrm{b} / \mathrm{b}}$ & $44.5 \pm 2.6^{b}$ \\
\hline G3 & $2.2 \pm 0.7$ & $9.2 \pm 4.6$ & $6.0 \pm 0.4^{\mathrm{c}}$ & $4.0 \pm 2.0$ & $5.3 \pm 0.9$ \\
\hline G4 & $0.4 \pm 0.2$ & $3.0 \pm 2.7$ & $3.4 \pm 2.3$ & $4.4 \pm 2.0$ & $4.8 \pm 0.9$ \\
\hline PI & $1.07 \pm 0.01$ & $1.21 \pm 0.035^{\mathrm{c}}$ & $1.206 \pm 0.05^{\mathrm{c}}$ & $1.29 \pm 0.01^{\mathrm{b} / \mathrm{b}}$ & $1.45 \pm 0.015^{\mathrm{c}}$ \\
\hline
\end{tabular}

${ }^{\mathrm{a}} \mathrm{EXO}$ or EXO/ev compared with PBS; IL-12 compared with EXO or EXO/ev; EXO/IL-12 compared with IL-12. ${ }^{\mathrm{b}} \mathrm{p}<0.05$, ${ }^{\mathrm{c}} \mathrm{p}<0.01$. G2, 3, 4: generation 2, 3, 4; PI, proliferation index.
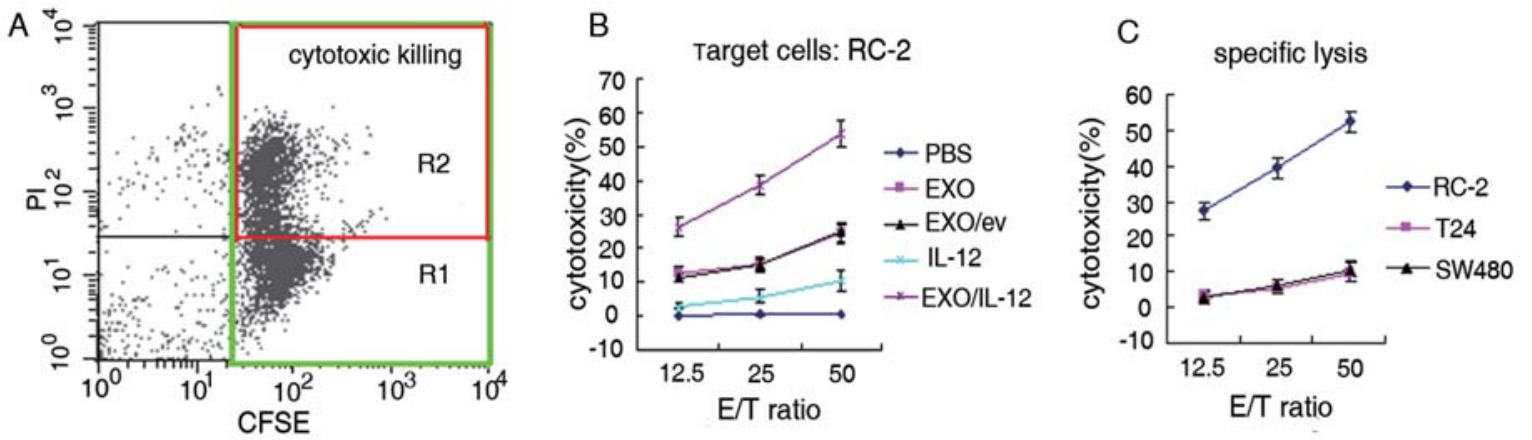

Figure 7. Increased induction of specific CTL activity by stimulation of EXO/IL-12. (A) A two-color flow cytometric assay measured cytotoxicity of tumor cells by effector cells. Lymphocytes were excluded by gating. Region 1 (R1, green) represents total $\mathrm{CFSE}^{+}$tumor cell targets. Region 2 (R2, red) contained target cells killed externally by effector cells (cytotoxicity) and were therefore CFSE $/ \mathrm{PI}^{+}$. Killing rate $=\left(\mathrm{CFSE}^{+} / \mathrm{PI}^{+}\right.$target cells) $/\left(\mathrm{CFSE}^{+}\right.$target cells). $(\mathrm{B})$ Lymphocytes were isolated from PBMCs and co-incubated with PBS, EXO, EXO/ev, IL-12 or EXO/IL-12 for $48 \mathrm{~h}$. The activated T cells used as effectors, and target cells were RC-2. Cytotoxicity assays based on PI costaining CFSE-labeled target cells were performed. (C) Cytotoxicity of T cells activated by EXO/IL-12 against three types of target cells (RC-2, T24 or SW480). Values are presented as mean \pm SD of triplicate wells.

$130 \pm 12.2 \mathrm{pg} / \mathrm{ml}$ secreted by lymphocytes induced by EXO and IL-12, respectively $(\mathrm{P}<0.01)$; the amount of IFN- $\gamma$ was $174.3 \pm 14.6 \mathrm{pg} / \mathrm{ml}$ secreted by lymphocytes induced by $\mathrm{EXO} / \mathrm{IL}-12$, which was more than that in the two other groups $(\mathrm{P}<0.05)$ (Fig. 6). These results indicated that $\mathrm{EXO} /$ IL-12 induce lymphocytes to secret IFN- $\gamma$ more powerfully than the conventional exosomes and IL- 12 .

More efficient induction of antigen-specific cytotoxic effect by exosomes GPI-IL-12. To explore whether EXO/IL-12 could elicit more potent cytotoxic effect, T cells were stimulated by PBS, EXO, EXO/ev, IL-12 or EXO/IL-12 loaded autologous dendritic cells for $48 \mathrm{~h}$, respectively, in five independent assays. Each groups had triplicate wells. Activated $\mathrm{T}$ cells used as effector cells were added to corresponding targets at varying effector-to-target cell ratios. The results (Fig. 7B) showed that initiation by EXO and EXO/IL-12 efficiently killed tumor cells as compared with PBS and IL-12 group $(\mathrm{P}<0.05)$; however, initiation by $\mathrm{EXO} / \mathrm{IL}-12$ exhibited the highest killing rate $(\mathrm{P}<0.01)$. To further explore whether EXO/IL-12 could induce antigen-specific cytotoxic effect, we used T-24, SW480 and RC-2 as target cells and evaluated the difference in killing rate. As shown in Fig. 7C, these results suggested that exosomes derived from renal cancer cell could activate $\mathrm{T}$ cells in antigen-specific manner through DCs, resulting in more efficient killing of renal cancer cells as compared with T-24 and sw480 cells. Taken together, these results implicated that IL-12-containg exosomes more efficiently induced antigen-specific cytotoxic effect than conventional exosomes.

\section{Discussion}

RCC constitutes 2-3\% of all solid malignancies and ranks 10th as the leading cause of death (1). Although surgery is the primary curative therapy for patients with local RCC, the prognosis for patients with advanced metastatic disease is poor, with a 5-year survival rate of $<10 \%$ (26). RCC is resistant to systemic chemotherapy and radiotherapy and is a good target for immunotherapy. In view of the observed spontaneous remissions of advanced RCC and infiltration of cancer tissue with dendritic cells and lymphocytes able to recognize malignant cells, immune mechanisms have been suggested to play a role in the natural disease course of RCC $(27,28)$. These findings led to attempts to develop novel immunotherapeutic strategies using vaccines. However, even if these new therapies look promising, the number of patients who are being cured remains frustratingly low. Ongoing efforts are still focused on optimizing vaccination strategies to produce curative antitumor immune responses.

An ideal tumor vaccine not only should be able to induce the production of tumor antigen-specific cytotoxic $\mathrm{T}$ 
lymphocytes (CTL) and strengthen Th1-related cell immune response, but also suppress Th2-related humoral immune response. However, this remains hampered by the lack of molecularly defined tumor antigen delivery or target systems (29). As a cell-free vaccine, tumor-derived exosomes, which are enriched in MHC-I, molecules, costimulatory molecules, heat shock protein, intercellular adhesion molecules and tumor-associated antigens, have met all the antigenpresenting conditions and effectively avoid the shortcomings of traditional vaccines. However, tumor-derived exosomes express certain immunosuppressive molecules, reducing their immunogenicity. FasL-expressing exosomes reduce T-cell responses by suppressing the expression of CD3- $\zeta$ and downregulating the JAK3-STAT5 signal pathway (12). TGFß1-expressing exosomes selectively impair lymphocyte response to interleukin-2 (30). Therefore, exosome-based immunotherapy needs to be modified to increase therapeutic efficacy. In this study, we prepared exosomes from IL-12anchored renal cancer cells and identified their morphology and protein composition, indicating that exosomes expressed immune-associated protein such as HSP70, ICAM-1, GPIIL-12 and RCC-associated antigen (G250), and IL-12 did not change the expression of these proteins. Therefore, the preparation of exosomes from fusion gene-modified renal cancer cells might be feasible for developing a good vaccine.

Systemic administration of IL-12 have been widely evaluated and showed extensive antitumor responses in murine models including various types of tumors (16). However, the potential use of IL-12 as immunomodulators in clinical practice has been hampered by its very short half-life and severe side effects of systemic administration. One of the ways to overcome this problem is to develop alternative approaches to deliver this cytokine locally or regionally. Protein transfer method is an alternative approach to deliver cytokines at the immunization site and express the desired cytokines for vaccine development (17). However, purified GPI-IL-12 protein must be obtained by protein transfer method, it is time-consuming and expensive. Furthermore, the activity of GPI-IL-12 is hard to maintain in the preparation procedure. In this study, we confirmed that exosomes isolated from IL-12anchored renal cancer cells also expressed GPI-IL-12. Firstly, the stable transfectants could be obtained from GPIIL-12 gene-modified renal cancer cells and IL-12 expressed on the cell surface via a GPI-moiety by confal laser scanning microscopy and flow cytometry. Secondly, IL-12 ELISA and Western blot analysis demonstrated that the fusion protein was also anchored on the exosomes derived from culture supernatants of IL-12 anchored RC-2 cells. The amount of IL-12 was $80 \pm 9.6 \mathrm{pg} / \mathrm{ml}$ in $10 \mu \mathrm{g}$ of exosomes. The mechanism of GPI-IL-12 spontaneously anchored onto exosomes was not clear. A possible reason is that GPI-IL-12 had existed on tumor cell membrane before exosomes formed by inward budding from the limiting cell membrane. Compared with protein transfer method, an alternative approach that we have developed to prepare exosomes containing GPI-IL-12 was easier and more efficient. Therefore, our results indicated that, by combining with the GPI anchor technology, EXO/ IL-12 could be prepared from IL-12-anchored renal cancer cells, and exosomes might serve as a valid vector to regionally deliver cytokines.
The antitumor immune response initiated by IL-12 has been attributed to the ability of this cytokine to induce IFN- $\gamma$ mediated activation of innate immunity (31), an antiangiogenic property (32), and the up-regulation of chemokines and adhesion molecules in tumor tissues (33) as well as, most important, its ability to promote the development of a Th1-type cellular immune response (15). Previous studies demonstrated that GPI-IL-12 induced the proliferation of activated T cells. Furthermore, GPI-IL-12 expressed on cells from various types of human tumors induced IFN- $\gamma$ release by activated T cells (17). In the present study, we have investigated whether EXO/IL-12 could also induce the proliferation and the secretion of IFN- $\gamma$ of activated T cells. As shown in Figs. 5 and 6, EXO/IL-12 significantly induced higher levels of secretion of IFN- $\gamma$ and proliferation of activated T cells as compared to exosomes and soluble IL-12. The reason for this difference between soluble IL-12 and EXO/IL-12 in induction of IFN- $\gamma$ production is not clear. It is possible that EXO/ IL-12 efficiently provided much more activated signals (such as co-stimulatory signals) to induce the proliferation of T cells and release of IFN- $\gamma$ than soluble IL-12.

The biological functions of exosomes largely depend on the cell types from which they are derived. Tumor cellderived exosomes were enriched with HSP70, ICAM-1 and tumor-associated antigen etc., which could trigger an effective immune response, as found in our present study as well as by others $(6,8)$. We firstly demonstrated that exosomes derived from renal cancer cells expressed RCC-associated antigen G250. G250 is the most important molecular marker and excellent therapeutic target in kidney cancer to date, and has been extensively applied in peptide-based vaccines and DCsbased vaccines (3). Heat shock protein 70 (HSP70) and intercellular adhesion molecule-1 (ICAM-1) were also highly expressed on exosomes. HSP70 known as 'molecular chaperone' is a highly effective antitumor immune response inducer, which binds tumor antigens and participates in presenting tumor antigens to MHC molecules, causing specific immune response (34). Exosomes carrying high amounts of HSPs could be good candidates for cancer immunotherapy. ICAM-1 is a kind of adhesion molecule of the immunoglobulin superfamily. ICAM-1 binds with its ligand lymphocyte function-associated antigen to promote immune differentiation and development, mediate killing effect of lymphocytes and natural killer cells to target cells, regulate lymphocyte activation, proliferation, cytotoxicity and cytokine production (35). However, the TEX-induced antitumor immunity was less efficicent because of the expression of immunosuppressive molecules on TEXs. Herein, we prepared exosomes from IL-12 anchored renal cancer cells and compared the antitumor effects of EXO/IL-12 with exosomes. As shown in Fig. 7, EXO/IL-12 exhibited much more potent antitumor effect than conventional exosomes and IL-12 in vitro. The results showed that through GPI anchor, cytokines can be efficiently anchored on exosomes and functionally delivered regionally by fusion gene transfection of tumor cells from which they derived. At the same time, the difference of cytotoxic effect for RC-2, T24 and SW480 initiated by renal cancer-derived exosomes, showed that exosomes could induce antigen-specific immunity response. 
In conclusion, we have developed an alternative approach to prepare exosomes GPI-IL-12 from fusion gene-modified renal cancer cells. We prepared exosome-based vaccine for renal cell cancer, which bears RCC-associated antigen G250 and GPI-IL-12 with the increased immunogenicity. Immunization with this vaccine can induce antigen-specific immune response and CTL more efficiently, resulting in more significant cytotoxic effects in vitro. Therefore, our study provides evidence that exosome-based tumor vaccine might be a novel strategy for treatment of renal cell carcinoma.

\section{Acknowledgements}

We thank Professor T.C. He (Department of Molecular Oncology, Chicago University, IL, USA)) for his gift of pAdTraceTO4-IL-12B-GPI plasmid and helpful advice.

\section{References}

1. Mathew A, Devesa S, Fraumeni JF and Chow WH: Global increases in kidney cancer incidence: 1973-1992. Eur J Cancer Prev 11: 171-178, 2002.

2. Glaspy JA: Therapeutic options in the management of renal cell carcinoma. Semin Oncol 29: 41-46, 2002.

3. Wierecky J, Müller MR, Wirths S, et al: Immunologic and clinical responses after vaccinations with peptide-pulsed dendritic cells in metastatic renal cancer patients. Cancer Res 66: 5910-5918, 2006.

4. Atzpodien J, Kirchner H, Jonas U, et al: Interleukin-2 and interferon-based immunochemotherapy in advanced renal cell carcinoma: a prospectively randomized trial of the German Cooperative Renal Carcinoma. Chemoimmunotherapy Group (DGCIN). J Clin Oncol 22: 1188-1194, 2004.

5. Raposo G, Nijman HW, Stoorvogel W, Liejendekker R, Harding CV, Melief CJ and Geuze HJ: B lymphocytes secrete antigen-presenting vesicles. J Exp Med 183: 1161-1172, 1996.

6. Andre F, Schartz NE, Movassagh M, et al: Malignant effusions and immunogenic tumor-derived exosomes. Lancet 360: 295305, 2002.

7. Chaput N, Taïeb J, Schartz NE, André F, Angevin E and Zitvogel L: Exosome-based immunotherapy. Cancer Immunol Immunother 53: 234-239, 2004.

8. Wolfers J, Lozier A, Raposo G, et al: Tumor-derived exosomes are a source of shared tumor rejection antigens for CTL crosspriming. Nat Med 7: 297-303, 2001.

9. Théry C, Zitvogel L and Amigorena S: Exosomes: composition, biogenesis and function. Nat Rev Immunol 2: 569-579, 2002.

10. Dai S, Wan T, Wang B, et al: More efficient induction of HLA$\mathrm{A}^{*}$ 0201-restricted and carcinoembryonic antigen (CEA)-specific CTL response by immunization with exosomes prepared from heat-stressed CEA-positive tumor cells. Clin Cancer Res 11: 7554-7563, 2005.

11. Zhang HG, Kim H, Liu C, et al: Curcumin reverses breast tumor exosomes mediated immune suppression of NK cell tumor cytotoxicity. Biochim Biophys Act 1773: 1116-1123, 2007.

12. Taylor DD and Gerçel TC: Tumour-derived exosomes and their role in cancer-associated T-cell signalling defects. Br J Cancer 92: 305-311, 2005.

13. Trinchieri G: Interleukin-12: a cytokine produced by antigenpresenting cells with immunoregulatory functions in the generation of T-helper cells type 1 and cytotoxic lymphocytes. Blood 84: 4008-4027, 1994.

14. Lesinski GB, Badgwell B, Zimmerer J, Crespin T, Hu Y, Abood G and Carson WE: IL-12 pretreatments enhance IFN-alphainduced Janus kinase-STAT signaling and potentiate the antitumor effects of IFN-alpha in a murine model of malignant melanoma. J Immunol 172: 7368-7376, 2004.
15. Colombo MP and Trinchieri G: Interleukin-12 in anti-tumor immunity and immunotherapy. Cytokine Growth Factor Rev 13: 155-168, 2002.

16. Sangro B, Melero I, Qian C, et al: Gene therapy of cancer based on interleukin 12. Curr Gene Ther 5: 573-581, 2005.

17. Nagarajan S and Selvaraj P: Glycolipid-anchored IL-12 expressed on tumor cell surface induced antitumor immune response. Cancer Res 62: 2869-2874, 2002.

18. Ji J, Li J, Holmes LM, Burgin KE, Yu X, Wagner TE and Wei Y: Glycoinositol phospholipid-anchored interleukin 2 but not secreted interleukin 2 inhibits melanoma tumor growth in mice. Mol Cancer Ther 1: 1019-1024, 2001.

19. Shuch B, Li Z and Belldegrun AS: Carbonic anhydrase IX and renal cell carcinoma: prognosis, response to systemic therapy, and future vaccine strategies. BJU Int 101: 25-30, 2008.

20. Lamparski HG, Metha-Damani A, Yao JY, Patel S, Hsu DH, Ruegg $\mathrm{C}$ and Le Pecq JB: Production and characterization of clinical grade exosomes derived from dendritic cells. J Immunol Methods 270: 211-226, 2002.

21. Zitvogel L, Regnault A, Lozier A, et al: Eradication of established murine tumors using a novel cell-free vaccine: dendritic cell-derived exosomes. Nat Med 4: 594-600, 1998.

22. Wang YC, Zhu L, McHugh R, et al: Induction of autologous tumor-specific cytotoxic T-lymphocyte activity against a human renal carcinoma cell line by B7-1 (CD80) costimulation. J Immunother 19: 1-8, 1996.

23. Lyons AB and Parish CR: Determination of lymphocyte division by flow cytometry. J Immunol Methods 171: 131-137, 1994.

24. Markel G, Seidman R, Cohen Y, et al: Dynamic expression of protective CEACAM1 on melanoma cells during specific immune attack. Immunology 126: 186-200, 2008.

25. Hao S, Moyana T and Xiang J: Cancer immunotherapy by exosome-based vaccines. Cancer Biother Radiopharmaceut 22: 692-703, 2007.

26. Kommu S: Renal-cell carcinoma: vaccination and risk of tumour progression. Lancet 363: 1557, 2004.

27. Belldegrun A, Muul LM and Rosenberg SA: Interleukin 2 expanded tumor-infiltrating lymphocytes in human renal cell cancer: isolation, characterization, and antitumor activity. Cancer Res 48: 206-214, 1988.

28. Thurnher M, Radmayr C, Ramoner R, et al: Human renal cell carcinoma tissue contains dendritic cells. Int J Cancer 68: 1-7, 1996.

29. Yang Y, Xiu F, Cai Z, Wang J, Wang Q, Fu Y and Cao X: Increased induction of antitumor response by exosomes derived from interleukin-2 gene-modified tumor cells. J Cancer Res Clin Oncol 133: 389-399, 2007.

30. Clayton A, Mitchell JP, Court J, Mason MD and Tabi Z: Human tumor-derived exosomes selectively impair lymphocyte responses to interleukin-2. Cancer Res 57: 7458-7466, 2007.

31. Smyth MJ, Taniguchi M and Street SE: The anti-tumor activity of IL-12: mechanisms of innate immunity that are model and dose dependent. J Immunol 165: 2665-70, 2000.

32. Gee MS, Koch CJ, Evans SM, et al: Hypoxia-mediated apoptosis from angiogenesis inhibition underlies tumor control by recombinant interleukin 12. Cancer Res 59: 4882-4889, 1999.

33. Fujiwara $\mathrm{H}$ and Hamaoka $\mathrm{T}$ : Coordination of chemokine and adhesion systems in intratumoral $\mathrm{T}$ cell migration responsible for the induction of tumor regression. Int Immunopharmacol 1: 613-623, 2001.

34. Gastpar R, Gehrmann M, Bausero MA, Asea A, Gross C, Schroeder JA and Multhoff G: Heat shock protein 70 surfacepositive tumor exosomes stimulate migratory and cytolytic activity of natural killer cells. Cancer Res 65: 5238-5247, 2005.

35. Segura E, Nicco C, Lombard B, et al: AM-1 on exosomes from mature dendritic cells is critical for efficient naive T-cell priming. Blood 106: 216-223, 2005. 